\section{Capnocytophaga bacteremia precipitating severe thrombo- cytopenia and preterm labor in an asplenic host}

\author{
Austin M. Hopkins, ${ }^{1}$ \\ Nerlyne Desravines, ${ }^{2}$ \\ Elizabeth M. Stringer, ${ }^{3}$ Katelin Zahn, ${ }^{2}$ \\ Carolyn M. Webster, ${ }^{3}$ Kayla Krajick, ${ }^{1}$ \\ Neeta L. Vora ${ }^{3}$ \\ ${ }^{1}$ University of North Carolina School of \\ Medicine, Chapel Hill, NC; ${ }^{2}$ Department \\ of Obstetrics \& Gynecology, University \\ of North Carolina School of Medicine, \\ Chapel Hill, NC; ${ }^{3}$ Division of Maternal \\ Fetal Medicine, Department of \\ Obstetrics \& Gynecology, University of \\ North Carolina School of Medicine, \\ Chapel Hill, NC, USA
}

\begin{abstract}
Capnocytophaga species are gram-negative bacilli that inhabit mammalian oral surfaces and can cause opportunistic infection, especially in asplenic patients. The species Capnocytophaga canimorsus is particularly associated with dog bites and is known to cause endocarditis, meningitis, and sepsis in the general population. In pregnant patients, infections tied to Capnocytophaga species from human flora have been associated with preterm labor, chorioamnionitis, and neonatal septicemia. There is little known about the effects of zoonotically-acquired Capnocytophaga infection in pregnant patients. In this case report, we present a patient with Capnocytophaga bacteremia acquired after a dog bite associated with profound thrombocytopenia and preterm labor. Dog bites are common in the United States, and we present basic recommendations for management of dog bites in pregnant patients in order to avoid morbidity associated with delay in time to antibiotic treatment of infection as described in this case.
\end{abstract}

\section{Introduction}

Capnocytophaga spp. are gram-negative bacilli that are colonized in mammalian oral flora but may also cause infection following dog or cat bites. ${ }^{1}$ Capnocytophaga canimorsus, (C. canimorsus), is a component of canine and feline oral flora and infection with $C$. canimorsus is primarily associated with dog bites. ${ }^{2}$ C. canimorsus is known to cause infections such as endo- carditis and meningitis and is associated with bacteremia and sepsis. ${ }^{3}$ People who are at increased risk of developing infections with $C$. canimorsus are asplenic patients and those who chronically use alcohol and considered functionally immunocompromised. ${ }^{4,5}$ Infection in pregnancy is rare however, the few case reports published have described premature labor, chorioamnionitis, and neonatal septicemia associated with C. ochracea and other species commonly found in human oral flora. ${ }^{6-9}$ However, little is known about the influence of zoonoticacquired Capnocytophaga infection during pregnancy.

\section{Case Report}

A 35-year-old female (gravida 5, para 2022) presented to a tertiary care hospital at 36 weeks and 2 days gestation with newonset vaginal bleeding and a five-day history of fevers, headache, neck stiffness, and altered mental status three days after being bitten by a neighbor's dog. Her prenatal history was significant for fetal intrauterine growth restriction (IUGR) diagnosed at $32 \mathrm{w} 5 \mathrm{~d}$ with a fetus weighing $1477 \mathrm{~g}$ ( $5^{\text {th }}$ percentile). Her past medical history was significant for a motor vehicular accident in a prior pregnancy with multiple traumatic injuries and resultant splenectomy. She also suffered a diffuse axonal brain injury with loss of short-term memory with a secondary seizure disorder. In subsequent pregnancies, she required a cesarean delivery due to an unstable pelvis. Prior to admission, she reported tremulous chills and fevers to $102.7^{\circ} \mathrm{F}\left(39.3^{\circ} \mathrm{C}\right)$. Her husband noted the patient was off-balance and was slurring her words. She also endorsed new-onset significant fatigue, persistent headache, and transient spotting across bilateral visual fields. The patient then began to experience new bright red vaginal bleeding. These symptoms prompted the patient's husband to bring her to the tertiary care hospital system 1.5 hours away from their home. The wound on her index finger from the dog bite appeared to be well-healing with no erythema, swelling, or tenderness to palpation.

Immediately on presentation, she was noted to have a persistently category 2 fetal heart tracing which was initially attributed to a placental abruption given her vaginal bleeding. She underwent an emergent repeat low transverse cesarean section. The neonate was admitted to the neonatal intensive care unit (NICU) and was diagnosed with respiratory failure necessitating intubation. and core temperature lability with stable hemodynamic vitals.

This patient's platelet count on admis-
Correspondence: Austin Hopkins, 5511 Butterfly Lane, Apt 302, Durham NC 27707 , USA.

Tel: +1.919.271.3811.

E-mail: austin_hopkins@med.unc.edu

Key words: Capnocytophaga, thrombocytopenia, preterm labor.

Acknowledgments: The authors would like to thank the staff of the Maternal Fetal Medicine service for their assistance in the care of the patient presented.

Contributions: $\mathrm{AH}$, manuscript writing, case analysis, and literature review; ND, manuscript review and case analysis; EMS, manuscript review and case analysis; KZ, manuscript review and case analysis; CMW, case analysis; KK, case analysis; NVL, manuscript review, case analysis.

Conflict of interest: The authors declare no potential conflict of interest.

Funding: None.

Received for publication: 7 August 2019.

Revision received: 23 October 2019.

Accepted for publication: 28 October 2019.

This work is licensed under a Creative Commons Attribution-NonCommercial 4.0 International License (CC BY-NC 4.0).

C Copyright: the Author(s), 2019

Licensee PAGEPress, Italy

Infectious Disease Reports 2019; 11:8272 doi:10.4081/idr.2019.8272

sion was $8 \times 10^{9} / \mathrm{L}$, which was confirmed with repeat testing. She was transfused prior to surgery with 2 units of platelet and responded appropriately with a count of $55 \times 10^{9} / \mathrm{L}$. In the immediate post-operative period, the patient's platelet count decreased to $16 \times 10^{9} / \mathrm{L}$, suggesting a thrombophilia. Routine laboratory screening during her pregnancy established a baseline platelet count of $400 \times 10^{9} / \mathrm{L}$. Initially, there was a concern for immune thrombocytopenia (ITP) or thrombotic thrombocytopenia (TTP), a hematologic emergency requiring plasmapheresis. However, the clinical history in this patient suggested an infectious etiology and splenic platelet sequestration impossible given the patient's lack of a spleen.

Hematology evaluation was negative for schistocytes on peripheral smear and revealed a normal ADAMST13 level, the latter of which essentially excluded TTP. However atypical, sepsis with or without disseminated intravascular coagulation (DIC) became the most likely underlying etiology. She had no evidence of DIC given 
normal coagulation studies including a normal fibrinogen, prothrombin time (PT) and partial prothrombin time (PTT). In sepsis syndromes, platelet counts generally are lower than $100 \times 10^{9} / \mathrm{L}$ with clinical severity associated with the magnitude of the decrease in platelet count, so this patient's profound thrombocytopenia due to infection was not a classic presentation. ${ }^{10}$ On further review, she had a remote history of a platelet count of $14 \times 10^{9} / \mathrm{L}$ around the time of the patient's traumatic MVA five years prior. ITP was an additional leading diagnosis for her thrombocytopenia that was unresponsive to transfusion, although the plausibility of profound platelet destruction by ITP in an asplenic patient was considered unlikely. Following cesarean delivery for category 2 fetal heart tracing on arrival, she was started on clindamycin, gentamicin, and metronidazole due to concern for an infectious process. The asplenic patient is an effectively immunocompromised patient that lacks cell-mediated immune defenses and are particularly susceptible to encapsulated organisms. ${ }^{11}$ In the setting of a dog bite, there is a higher risk of pathologic infection with canine oral flora, particularly Capnocytophaga. Bacteremia with Capnocytophaga is exceedingly rare but potentially serious, with case reports describing a range of $25 \%-60 \%$ mortality from sepsis secondary to $C$. canimorsus. ${ }^{12}$ Other considerations in this setting included rabies. Per the report of the patient's neighbor, the dog was fully vaccinated. After quarantine, the dog in question showed no evidence of rabies. On immediate evaluation following the dog bite at an urgent care facility, rabies prophylaxis had been deferred due to low suspicion of canine infection. The Infectious Disease specialists were consulted given the high risk for pathologic infection with an atypical organism. As part of a complete workup, she had an x-ray of the finger were unremarkable for acute signs of osteomyelitis. She was started on meropenem for antibiotic therapy, a carbapenem beta-lactam providing broad coverage against both gram positive and negative organisms. On a detailed exposure history, the patient was found to have tick bite in the preceding months as well as contact with goat placenta from goats that had recently given birth on her farm, a known harbinger of Coxiella burnetii, the common cause of Q fever. ${ }^{13}$ Given the patient's altered state may have been the result of exposure to tick-borne rickettsial diseases, she was also started on doxycycline.

Another difficulty in this thrombocytopenic patient was further evaluation of a meningeal process. She had neck stiffness and a fluctuating mental status suggesting an intracranial component to her symptoms. With her platelet count of less than $70 \times 10^{9} / \mathrm{L}$, a lumbar puncture placed her at high risk for bleeding. ${ }^{14}$ In discussion with the Maternal Fetal Medicine, Hematology, and Infectious Disease teams, the benefits and risks of this invasive procedure were discussed. The patient's symptomology was determined to be more suggestive encephalopathy, due to the altered mental status being the predominant neurological finding. Lumbar puncture was deferred. An MRI, in the evaluation of infectious central nervous system disease, is a sensitive form of neuroimaging. ${ }^{15}$ She underwent an MRI of the brain with contrast which showed no evidence of encephalitis, no acute intracranial processes but did note some chronic change consistent with her prior brain injury.

Blood cultures obtained at admission were positive for gram negative bacilli and preliminarily grew Capnocytophaga species and she was converted to IV ertapenem, a carbapenem betalactam antibiotic, for a 10-day course. Capnocytophaga canimorsus was eventually isolated from the cultures. Bacterial identification was accomplished via MALDITOF mass spectrometry with $16 \mathrm{~S}$ rRNA sequencing for verification. The neonate was sent to the NICU following delivery and was briefly intubated for respiratory distress, but otherwise had no complications and was discharged to the newborn nursery on the $6^{\text {th }}$ day of life. Blood cultures from the neonate were negative. With the administration of IV antibiotics, the patient's platelet count recovered to $278 \times 10^{9} / \mathrm{L}$ one day after starting the IV ertapenem. Her profound thrombocytopenia, despite not having a spleen, was attributed to platelet consumption and decreased platelet production due to $C$. canimorsus infection. Although the pathogenesis of thrombocytopenia in sepsis is largely unknown, there is some evidence that absolute immature platelet count (AIPC) can decrease past the acute phase of sepsis, suggesting thrombopoietic suppression and a subsequent decrease in platelet production. ${ }^{16}$ The patient was discharged on the sixth hospital day and completed IV ertapenem therapy on an outpatient basis at home.

\section{Discussion}

The patient's asplenia presented a clinical challenge. This differential diagnosis for the patient's clinical illness was particularly extensive given her co-morbidities. She had severe thrombocytopenia with an initial platelet count of $8 \times 10^{9} / \mathrm{L}$, suggesting a hematologic pathology. Concurrently, she presented with fevers and altered mental status following multiple zoonotic exposures. The impact of both processes was concerning particularly in the setting of intrauterine growth restriction.

\section{Fetal considerations}

Perinatal infection with Capnocytophaga species is rare with only scattered cases across medical literature with a predominance of focus on species found in human flora. Infection with these species are a demonstrated potential risk factor for preterm birth and an infrequent cause of chorioamnionitis and neonatal infection. ${ }^{17}$ Several reported cases involved afebrile women who presented in preterm labor with intact membranes and mild leukocytosis. ${ }^{18-21}$ Three cases described neonatal morbidity as

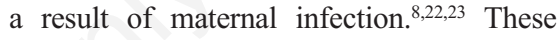
reports are based on relatively healthy mothers and could suggest that overall, infections from human flora Capnocytophaga species are relatively benign with low risk of longterm morbidity.

\section{Immunocompromised host}

Our case uniquely illustrates the potential gravity of zoonotic Capnocytophaga infection in an immunocompromised pregnant woman, especially in the setting of asplenia. C. canimorsus was isolated in this case and is the most common Capnocytophaga species associated with dog bites. ${ }^{2}$ C. canimorsus has a polysaccharide capsule that is implicated in resistance to the innate immunity of the host. ${ }^{22}$ One study showed that infection with $C$. canimorsus does not elicit an inflammatory response, and this is potentially due to the protective nature of a polysaccharide structure from deposition of the complement membrane attack complex and from efficient phagocytosis by polymorphonuclear leukocytes (PMNs). ${ }^{25,26}$ This may suggest that although the clinical presentation in a relatively healthy patient may be unremarkable, the underlying infection could still be severe, and the risk of infection on both maternal and neonatal morbidity during pregnancy could be increased with maternal immunocompromise. Our case illustrates an acutely ill patient who is immunocompromised at baseline. Although she was hemodynamically stable, our patient may have been in subclinical or atypical sepsis from C. canimorsus infection, as seen by fetal distress, dramatic maternal thrombocytopenia, labile fevers, central nervous system symptoms, and altered mental status. 


\section{Severe thrombocytopenia}

The profound thrombocytopenia in this case is of particular interest. Given the magnitude of the depression in platelet counts, thrombocytopenia in this case was almost certainly not attributable to gestational thrombocytopenia alone. ${ }^{14}$ Also, gestational thrombocytopenia is not associated with symptomatic patients and other findings such as this patient's altered mental status. Thrombotic microangiopathy was unlikely due to two peripheral smears negative for schistocytes..$^{27}$ The patient's relatively unremarkable ADAMTS13 result essentially excluded TTP, and ITP is not consistent with sudden rebound of platelet levels after antibiotic administration. DIC is unlikely as the patient's fibrinogen levels remained elevated throughout her course. A possible explanation is direct bone marrow suppression in the setting of fulminant infection or sepsis, although it is intriguing that the patient was never classically septic and that only the platelet cell line was remarkably depressed, as the patient's red blood cell counts were only slightly below normal range and her white blood cell counts were normal to elevated.

\section{Dog bite}

Animal bites account for $1 \%$ of all injury-related emergency department visits and incur more than $\$ 50$ million in inpatient care costs annually. ${ }^{28,29}$ Most animal bites are from dogs, with most animals being familiar to the victim. Antibiotic prophylaxis is recommended for patients suffering a dog bite, especially if the patient is immunosuppressed. Amoxicillin-clavulanate is the first-line antibiotic for treatment of dog bites, and is safe in pregnancy. ${ }^{30,31}$ Capnocytophaga infections are covered by amoxicillin-clavulanate, and early treatment of infection in our patient may have prevented the ensuing morbidity, including preterm labor and preterm delivery. ${ }^{32}$ Our patient reported being prescribed antibiotics to treat her dog bite but was instructed not to start her regimen until she was able to be seen by her regular $\mathrm{Ob} / \mathrm{Gyn}$. The delay in time to antibiotic treatment may have been the precipitous factor in her preterm labor and delivery.

\section{Conclusions}

Capnocytophaga canimorsus was identified in blood cultures after a dog bite in a pregnant patient with baseline immunocompromise due to asplenia. In this case, infection was associated with fetal distress, preterm labor, vaginal bleeding, severe maternal thrombocytopenia, and a brief period of neonatal respiratory distress requiring intubation, although the latter finding may be more specifically related to prematurity rather than a direct consequence of specific maternal infection. This case report suggests that both maternal and neonatal effects of $C$. canimorsus infection can be serious, especially with zoonoticacquired infections and with a delay in time to treatment. As effective and safe treatment exists, we recommend that all pregnant patients who present to any medical provider with dog bite be treated prophylactically with antibiotics, especially in patients with any form of immunocompromise. For symptomatic pregnant patients, evaluation in a labor and delivery triage unit for fetal monitoring may be necessary. The outcomes in this case were favorable, and this may be partly due to the arrival of the patient to a tertiary care hospital system, where infectious disease, hematological, and neonatal intensive care resources were plentiful and readily accessible. Further research is needed to better understand the mechanism of the profound, isolated depression of platelet counts during this patient's illness.

\section{References}

1. Brenner DJ, Hollis DG, Fanning GR, Weaver RE. Capnocytophaga canimorsus sp. nov. (formerly CDC group DF2), a cause of septicemia following dog bite, and C. cynodegmi sp. nov., a cause of localized wound infection following dog bit. J Clin Microbiol 1989;27:2315.

2. Lion C, Escande F, Burdin JC. Capnocytophaga canimorsus infections in humans: review of the literature and cases report. Eur J Epidemiol 1996;12: 521-33.

3. Zajkowska J, Krol M, Falkowski D, et al. Capnocytophaga canimorsus - an underestimated danger after dog or cat bite - review of literature. Przegl Epidermiol 2016;70:289-95.

4. Janda JM, Graves MH, Lindquist D, Probert WS. Diagnosing Capnocytophaga canimorsus infections. Emerg Infect Dis 2006;12:340-2.

5. Pers C, Gahrn-Hansen B, Frederiksen W Capnocytophaga canimorsus septicemia in Denmark, 1982-1995: review of 39 cases. Clin Infect Dis 1996;23:71-5.

6. Alhifany AA, Almangour TA, Tabb DE, Levine DH. Premature Labor and Neonatal Septicemia Caused by Capnocytophaga Ochracea. Am J Case
Rep 2017; 18:674-6.

7. Paerregaard A, Gutschik E. Capnocytophaga bacteremia complicating premature delivery by cesarean section. Eur J Clin Microbiol 1987;6:5801.

8. Mayatepek E, Zilow E, Pohl S. Severe intrauterine infection due to Capnocytophaga ochracea. Biol Neonate 1991;60:184-6.

9. Mekouar H, Voortman G, Bernard P, et al. Capnocytophaga species and perinatal infections: case report and review of literature. Acta Clin Belg 2012;67:42-5.

10. Claushuis T.A.M., van Vught L.A. Thrombocytopenia is associated with a dysregulated host response in critically ill sepsis patients. Blood 2016;127:3062-72.

11. Ram S, Lewis LA, Rice PA. Infections of People with Complement Deficiencies and Patients Who Have Undergone Splenectomy. Clin Microbiol Rev 2010;23:740-80.

12. Shahani L, Khardori N. Overwhelming Capnocytophaga canimorsus infection a patient with asplenia. BMJ Case Rep 2014;2014:bcr2013202768.

13. Roest HJ, van Gelderen B, Dinkla A, et al. Q fever in pregnant goats: pathogenesis and excretion of Coxiella burnetii. PLoS One 2012;7:e48948.

14. American College of Obstetricians and Gynecologists. Thrombocytopenia in pregnancy. ACOG Practice Bulletin No. 207. Obstet Gynecol 2019;133:e18193.

15. Maschke M, Kastrup O, Forsting M, Diener HC. Update on neuroimaging in infectious central nervous system disease. Curr Opin Neurol 2004;17:475.

16. Koyama K, Katayama S, Muronoi T, et al. Time course of immature platelet count and its relation to thrombocytopenia and mortality in patients with sepsis. PLoS One 2018;13:e192064.

17. Lopez E, Raymond J, Patkai J, et al. Capnotycophaga species and preterm birth: case series and review of the literature. Clin Microbiol Infect 2010:16:1539-43.

18. Ernest JM, Wasilauskas B. Capnocytophaga in the amniotic fluid of a woman in preterm labor with intact membranes. Am J Obstet Gynecol 1985;153:648-9.

19. McDonald H, Gordon DL. Capnocytophaga species: a cause of amniotic fluid infection and preterm labour. Pathology 1988;20:74-6.

20. Edwards C, Yi CH, Currie JL. Chorioamnionitis caused by Capnocytophaga: case report. Am J Obstet Gynecol 1995;173:244-5. 
21. Mercer LJ. Capnocytophaga isolated from the endometrium as a cause of neonatal sepsis. A case report. J Reprod Med 1985;30:67-8.

22. Feldman JD, Kontaxis EN, Sherman MP. Congenital bacteremia due to Capnocytophaga. Pediatr Infect Dis 1985;4:415-6.

23. Bass WT, DeLoach D, Khan JH, et al. Severe Cystic Pericentricular Leukomalacia in a Premature Infant with Capnocytophagia Sepsis. Am J Perinatol Rep 2014;4:e73-6.

24. Hess E, Renzi F, Koudad D, et al. Identification of Virulent Capnocytophaga canimorsus Isolates by Capsular Typing. J CLin Microbiol 2017;55:1902-14.

25. Shin H, Mally M, Kuhn M, et al. Escape from immune surveillance by Capnocytophaga canimorsus. J Infect Dis 2007;195:375086.

26. Shin H, Mally M, Meyer S, et al. Resistance of Capnocytophaga canimorsus to killing by human complement and polymorphonuclear leukocytes. Infect Immun 2009;77:2262-71.

27. Reese JA, Peck JD, Deschamps DR, et al. Platelet counts during pregnancy. N Engl J Med 2018;379:32.

28. Holmquist L, Elixhauser A. Emergency department visits and inpatient stays involving dog bites, 2008. November 2010. Rockville, Md.: Agency for Healthcare Research and Quality; 2010. http://www.hcup-us.ahrq.gov/reports/ statbriefs/sb101.pdf. Accessed June 24, 2014.
29. Centers for Disease Control and Prevention. Nonfatal dog bite-related injuries treated in hospital emergency departments-United States, 2001. MMWR Morb Mortal Wkly Rep 2003;52:605-10.

30. Ellis R, Ellis C. Dog and cat bites. Am Fam Physician 2014;90:239-43.

31. Muanda FT, Sheehy O, Berard A. Use of antibiotics during pregnancy and the risk of major congenital malformations: a population based cohort study. Br J Clin Pharmacol 2017;83:2557-71.

32. Jolivet-Gougeon A, Sixou JL, TamanaiShacoori Z, Bonnaure-Mallet M. Antimicrobial treatment of Capnocytophaga infections. Int $\mathrm{J}$ Antimicrob Agents 2007;29:367-73. 